\title{
THE RECIPROCITY PRINCIPLE FOR A NONLINEAR ANISOTROPIC MEDIUM WITHOUT HYSTERESIS: THEORY AND PRACTICE OF APPLICATION
}

The construction of the correct vector material equations for nonlinear anisotropic soft magnetic materials remains one of the main reserves for increasing the accuracy of mathematical models in solving magnetostatic problems in the field formulation. The aim of the work is to establish asymptotic expressions for the reciprocity principle, which is a fundamental property of reversible magnetization processes of nonlinear anisotropic media, and to use the obtained results to optimize the computational process when constructing the vector magnetization characteristic and differential permeability tensor. The potentiality property of the magnetic flux density vector $B$ in $\mathbf{H}$-space is used. The main result of the paper is an illustration, using concrete examples, of an alternative method for calculating vector magnetization characteristics for one of the orthogonal families. In order to eliminate the instrumental error and ensure maximum accuracy and reliability of the obtained results, the exact characteristics for the components of the vector magnetization characteristic obtained by differentiating a special analytical expression for the potential were used as initial ones. The principle of reciprocity, by virtue of its universal nature, makes a significant contribution to the theory of nonlinear anisotropic media in the hysteresis-free approximation. Asymptotic expressions for the reciprocity principle are obtained for the first time. The performed computational experiments on the construction of vector characteristics based on the known magnetization characteristics in one of the directions confirm almost complete coincidence with the exact values obtained analytically. The use of asymptotic expressions for the reciprocity principle not only greatly simplifies computational processes for determining the orthogonal magnetization characteristics, but also implements the calculation of differential permeability tensors for arbitrary field values. The proposed method can be implemented in applications for calculating the magnetic field in devices with nonlinear anisotropic magnetically soft materials, primarily with cold rolled sheet electrical steels, which are most used in electrical engineering. References 12, figures 5.

Key words: nonlinear anisotropic medium, vector magnetization characteristics, energy potential, reciprocity principle, asymptotic expressions, magnetic permeability tensor.

Розглянуто теоретичні та практичні аспекти побудови векторних матеріальних рівнянь нелінійних анізотропних середовищ. Показано, що існуючі методи обліку магнітних властивостей навіть в безгістерезисному наближенні не завжди задовольняють вимогам повноти й математичної строгості. Підтверджено ефективність енергетичного підходу до побудови векторних характеристик магнітного стану таких середовищ. Особливу увагу приділено принципу взасмності як фундаментальній властивості оборотних процесів намагнічування. Встановлено нові асимптотичні вирази для принципу взаємності і на численних прикладах показана їх ефективність при побудові векторної моделі магнітного середовища без використання енергетичного потенціалу. Бібл. 12, рис. 5.

Ключові слова: нелінійне анізотропне середовище, векторні характеристики намагнічування, енергетичний потенціал, принцип взаємності, асимптотичні вирази, тензор магнітної проникності.

Рассмотрены теоретические и практические аспекты построения векторных материальных уравнений нелинейных анизотропных сред. Показано, что используемые методы учета магнитных свойств даже в безгистерезисном приближении не всегда удовлетворяют требованиям полноты и математической строгости. Подтверждена эффективность энергетического подхода к построению векторных характеристик магнитного состояния таких сред. Особое внимание уделено принципу взаимности как фундаментальному свойству обратимых процессов намагничивания. Установлены новые асимптотические выраэжения для принципа взаимности и на численных примерах показана их эффективность при построении векторной модели магнитной среды без использования энергетического потенциала. Библ. 12, рис. 5.

Ключевые слова: нелинейная анизотропная среда, векторные характеристики намагничивания, энергетический потенциал, принцип взаимности, асимптотические выражения, тензор магнитной проницаемости.

Introduction. Advances in information technology in recent decades have stimulated the development of methods for mathematical modelling of magnetic fields in various electrophysical devices [1-7]. One of the important stages of the practical implementation of problems in the field formulation is the formation of the material equations of the magnetic medium, which in most cases has nonlinear anisotropy. The completeness of the taking into account of magnetic properties of such a medium requires the construction of a vector characteristic $\boldsymbol{B}(\boldsymbol{H})$. The construction of vector models based on the scalar dependencies $B_{i}\left(H_{i}\right)$ for the principal axes of anisotropy [3, 4], the taking into account of magnetic anisotropy in the framework of «elliptic models» $[4,5]$, and other simplified approaches are more likely forced than constructive solution and, as shown in
[8], inevitably lead to a loss of information and an almost uncontrolled calculation error.

Some progress in taking into account of the nonlinear anisotropy of the vector models $\boldsymbol{B}(\boldsymbol{H})$ in the hysteresis-free approximation is associated with the energy approach [1, 6-10]. The basic relation of this approach is the expression [11]

$$
\oint_{L_{H}} \boldsymbol{B} \cdot \mathrm{d} \boldsymbol{H}=0,
$$

where $L_{\boldsymbol{H}}$ is the arbitrary closed contour in $\boldsymbol{H}$-space, i.e. in space, on the axes of which the quantities $H_{1}$ and $H_{2}$ are plotted and which in essence is the hodograph of the vector $\boldsymbol{H}$ with an arbitrary cyclic magnetization reversal of the medium. With such a definition of $\boldsymbol{H}$-space,

(C) S.T. Tolmachev, A.V. Il'chenko 
relation (1) formally coincides with the classical condition of potentiality, since an expression under the sign of the integral is the total differential of the potential $\Psi(\boldsymbol{H})$.

The above definition of $\boldsymbol{H}$-space indicates the independence of the potential $\Psi(\boldsymbol{H})$ from the integration path in $\boldsymbol{H}$-space, the eddy-free character of the magnetic flux density field $\boldsymbol{B}$, which is the force vector of the field in this space:

$$
\boldsymbol{B}=-\frac{\mathrm{d} \psi}{\mathrm{d} \boldsymbol{H}}=\operatorname{grad}_{\boldsymbol{H}} \Psi(\boldsymbol{H})=\boldsymbol{i}_{1} B_{1}\left(H_{1}, H_{2}\right)+\boldsymbol{i}_{2} B_{2}\left(H_{1}, H_{2}\right) .
$$

In addition, we note an important consequence of relations (1) and (2) - the symmetry of the differential magnetic permeability tensor $\boldsymbol{\mu}_{\mathrm{d}}(\boldsymbol{H})$ :

$$
\boldsymbol{\mu}_{\mathrm{d}}=\frac{\mathrm{d} \boldsymbol{B}}{\mathrm{d} \boldsymbol{H}} ; \quad \mu_{\mathrm{d} i j}=\frac{\partial^{2} \Psi}{\partial H_{i} \cdot \partial H_{j}}=\frac{\partial^{2} \Psi}{\partial H_{j} \cdot \partial H_{i}}=\mu_{\mathrm{d} j i} .
$$

In formulas (2), (3), we use the notation associated with the concepts of the derivative of a scalar and a vector function with respect to the vector argument used in vector algebra [12, items 6.2, 6.3].

Energy potentials cannot be measured directly; therefore, their construction is a difficult problem even in the two-dimensional case [7]. The basic information for construction of the potential is a certain set of experimentally measured magnetization characteristics, which are used either by their direct integration, or by selecting the coefficients of some analytical dependence $[7,8]$. Leaving beyond the scope of this paper the known problems with the accuracy of measuring the magnetic characteristics of anisotropic materials, we note the obvious problems with the numerical differentiation of the potential $\Psi(\boldsymbol{H})$ : direct - to obtain the vector dependence $\boldsymbol{B}(\boldsymbol{H})$ and repeated - for the differential magnetic permeability tensor $\boldsymbol{\mu}_{\mathrm{d}}(\boldsymbol{H})$.

An alternative way to determine the magnetization characteristics in mutually orthogonal directions without explicitly defining the potential $\Psi(\boldsymbol{H})$ is the reciprocity principle, first formulated in [9] and developed in a number of subsequent publications (see, for example, $[8,10])$. The reciprocity principle is a fundamental property of an anisotropic medium without hysteresis and is based on the independence of the potential $\Psi(\boldsymbol{H})$ from the integration path in $\boldsymbol{H}$-space.

Let $H_{1}^{\prime} \leq H_{1} \leq H_{1}^{\prime \prime}$ and $H_{2}^{\prime} \leq H_{2} \leq H_{2}^{\prime \prime}$. Equation (1) implies the equality of the integrals $S_{1}$ and $S_{2}$ :

$$
\begin{aligned}
& S_{1}=\int_{H_{1}^{\prime}}^{H_{1}^{\prime \prime}}\left[B_{1}\left(H_{1}, H_{2}^{\prime}\right)-B_{1}\left(H_{1}, H_{2}^{\prime \prime}\right)\right] \cdot \mathrm{d} H_{1} ; \\
& S_{2}=\int_{H_{2}^{\prime}}^{H_{2}^{\prime \prime}}\left[B_{2}\left(H_{1}^{\prime}, H_{2}\right)-B_{2}\left(H_{1}^{\prime \prime}, H_{2}\right)\right] \cdot \mathrm{d} H_{2} .
\end{aligned}
$$

Energy relations (4) have a simple geometric meaning: the areas of the corresponding curved quadrangles $S_{1}$ and $S_{2}$ are the same. Visually, relations (4) at $H_{1}^{\prime}=H_{2}^{\prime}=0$ and $H_{1}^{\prime \prime}=H_{1}^{*}, \quad H_{2}^{\prime \prime}=H_{2}^{*}$ are shown in Fig. 1.
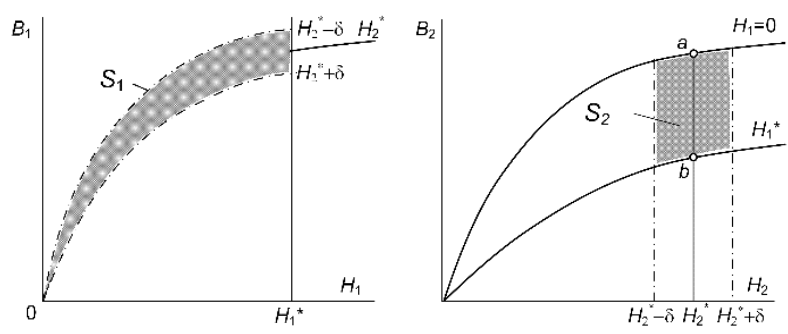

Fig. 1. Geometric meaning of the principle of reciprocity

In practical terms, the importance of the reciprocity principle lies in the possibility of constructing a vector magnetization characteristic $\boldsymbol{B}(\boldsymbol{H})$ from partially given information about the magnetic properties of a nonlinear anisotropic medium.

The goal of this paper is further generalization of the reciprocity principle (4), in particular, obtaining its asymptotic expressions and using them to optimize the computational process when constructing the vector characteristic $\boldsymbol{B}(\boldsymbol{H})$ and the differential magnetic permeability tensor $\boldsymbol{\mu}_{\mathrm{d}}(\boldsymbol{H})$. As we know, it is this information about the magnetic properties of the medium that is used in various computational schemes. reciprocity.

Asymptotic expressions for the principle of

A. The case of Cartesian coordinates. We write relations (4) in relation to Fig. 1:

$$
\begin{aligned}
& S_{1}=\int_{0}^{H_{1}^{*}}\left[B_{1}\left(H_{1}, H_{2}^{*}+\delta\right)-B_{1}\left(H_{1}, H_{2}^{*}-\delta\right)\right] \cdot \mathrm{d} H_{1} ; \\
& S_{2}=\int_{H_{2}^{*}-\delta}^{H_{2}^{*}+\delta}\left[B_{2}\left(0, H_{2}\right)-B_{2}\left(H_{1}^{*}, H_{2}\right)\right] \cdot \mathrm{d} H_{2} .
\end{aligned}
$$

Since relations (5) are valid for arbitrary values of $\delta$, we consider the limiting expressions for the integrals $R_{1}^{*}=R_{2}^{*}$ :

$$
\begin{aligned}
& R_{1}^{*}=\lim _{\delta \rightarrow 0} \int_{0}^{H_{1}^{*}} \frac{1}{2 \delta}\left[B_{1}\left(H_{1}, H_{2}^{*}+\delta\right)-B_{1}\left(H_{1}, H_{2}^{*}-\delta\right)\right] \cdot \mathrm{d} H_{1} ; \\
& R_{2}^{*}=\lim _{\delta \rightarrow 0} \int_{H_{2}^{*}-\delta}^{H_{2}^{*}+\delta} \frac{1}{2 \delta}\left[B_{2}\left(0, H_{2}\right)-B_{2}\left(H_{1}^{*}, H_{2}\right)\right] \cdot \mathrm{d} H_{2} .
\end{aligned}
$$

For small values of $\delta$, the integrand in the first integral (6) can be expressed in terms of differential magnetic permeability $\mu_{\mathrm{d} 12}$ :

$$
\lim _{\delta \rightarrow 0}\left[\Delta B_{1}\left(H_{1}, H_{2}^{*}\right) / \Delta H_{2}\right] \approx \mu_{\mathrm{d} 12}\left(H_{1}, H_{2}^{*}\right),
$$

therefore, the integral $R_{1}^{*}$ becomes curved and takes the form

$$
R_{1}^{*}=\int_{0}^{H_{1}^{*}} \mu_{\mathrm{d} 12}\left(H_{1}, H_{2}^{*}\right) \cdot \mathrm{d} H_{1} .
$$

As for the integral $R_{2}^{*}$, as can be seen from Fig. 1, at $\delta \rightarrow 0$, the area $S_{2}$ degenerates into a line $a b$, which corresponds to the increment of the magnetic flux density component $B_{2}$ at $H_{2}=H_{2}^{*}$ and $0 \leq H_{1} \leq H_{1}^{*}$. Therefore 


$$
R_{2}^{*} \rightarrow a b=\int_{0}^{H_{1}^{*}} \mu_{\mathrm{d} 12}\left(H_{1}, H_{2}^{*}\right) \cdot \mathrm{d} H_{1} .
$$

Obviously, when the integration limits are compressed to a point, we obtain the symmetry of the differential magnetic permeability tensor $\boldsymbol{\mu}_{\mathrm{d}}(\boldsymbol{H})$ established previously by relation (3). Thus, the reciprocity principle has three possible representations: point one (at each «point» $\boldsymbol{H})-(3)$ and two integral ones - respectively (4) and (7), (8).

We note the important practical significance of the obtained asymptotic expressions. It was shown in [8] that the restoration of potential $\Psi$ is possible from a family of magnetization characteristics in one direction, for example, $B_{1}\left(H_{1}, H_{2}\right)$ and one orthogonal characteristic, for example, $B_{2}\left(0, H_{2}\right)$. As will be shown below, the missing array of points of characteristics $B_{2}\left(H_{1}, H_{2}\right)$ can be obtained without calculating the potential $\Psi$ using expression (8).

Note that by rearranging the indices, we can obtain relations similar to (7), (8) for other initial data, for example, $B_{2}\left(H_{1}, H_{2}\right)$ and $B_{1}\left(H_{1}, 0\right)$ :

$$
\begin{gathered}
R_{2}^{*}=\int_{0}^{H_{2}^{*}} \mu_{\mathrm{d} 21}\left(H_{2}, H_{1}^{*}\right) \cdot \mathrm{d} H_{2} ; \\
R_{1}^{*} \rightarrow c d=\int_{0}^{H_{2}^{*}} \mu_{\mathrm{d} 12}\left(H_{2}, H_{1}^{*}\right) \cdot \mathrm{d} H_{2},
\end{gathered}
$$

and $R_{1}^{*}=R_{2}^{*}$.

B. The case of polar coordinates. Relations (1), (2) are invariant with respect to the coordinate system. We assume that the family of «longitudinal» magnetization characteristics $B_{\|}(H, \alpha)$, where $B_{\|}$is the projection of the vector $\boldsymbol{B}$ onto the vector $\boldsymbol{H}$, and $\alpha$ is the angle defining the direction of the vector $\boldsymbol{H}$ is specified (basic) information. For the vector $\boldsymbol{B}$, we use the decomposition $\boldsymbol{B}=\boldsymbol{B}_{\|}(H, \alpha)+$ $+\boldsymbol{B}_{\perp}(H, \alpha)$, where $\boldsymbol{B}_{\perp}(H, \alpha)$ is the family of characteristics of «transverse» magnetization orthogonal to $\boldsymbol{B}_{\|}(H, \alpha)$.

In view of the above, formula (2) takes the form

$$
\boldsymbol{B}=\frac{\mathrm{d} \Psi}{\mathrm{d} \boldsymbol{H}}=\operatorname{grad}_{\boldsymbol{H}} \Psi(\boldsymbol{H})=\boldsymbol{r}^{0} \frac{\partial \Psi}{\partial H}+\boldsymbol{\alpha}^{0} \frac{1}{H} \cdot \frac{\partial \Psi}{\partial H},
$$

where $r^{0}, \alpha^{0}$ are the unit vectors of the polar coordinate system.

We note the possibility of reconstructing the potential from a given family of characteristics $\boldsymbol{B}_{\|}(H, \alpha)$. So, taking $\Psi(0)=0$, for an arbitrary point $\boldsymbol{H}=\left(H, \alpha^{*}\right)$ we obtain

$$
\Psi(\boldsymbol{H})=\int_{0}^{H} B_{\|}(H, \alpha) \cdot \mathrm{d} H
$$

and $B_{\perp}(H, \alpha)=\frac{1}{H} \cdot \frac{\partial \Psi}{\partial \alpha}$.

As in the case of Cartesian coordinates, to calculate the orthogonal characteristics $B_{\perp}(H, \quad \alpha)$ without calculating the potential, we use the reciprocity principle.
Let $H^{\prime} \leq H \leq H^{\prime \prime}$ and $\alpha^{\prime} \leq \alpha \leq \alpha^{\prime \prime}$. We have the equality of integrals $S_{1}$ and $S_{2}$ :

$$
\begin{aligned}
& S_{1}=\int_{H^{\prime}}^{H^{\prime \prime}}\left[B_{\|}\left(H, \alpha^{\prime}\right)-B_{\|}\left(H, \alpha^{\prime \prime}\right)\right] \cdot \mathrm{d} H ; \\
& S_{2}=\int_{\alpha^{\prime}}^{\alpha^{\prime \prime}}\left[H^{\prime} \cdot B_{\perp}\left(H^{\prime}, \alpha\right)-H^{\prime \prime} \cdot B_{\perp}\left(H^{\prime \prime}, \alpha\right)\right] \cdot \mathrm{d} \alpha .
\end{aligned}
$$

The proof and illustration of relations (12) are given in [9]. We establish the asymptotic properties of the reduced integral reciprocity principle, similar to relations (9) and (10). Let $\boldsymbol{H}^{*}=\left(H^{*}, \alpha^{*}\right)$ be given - some point in the intervals $H^{\prime} \leq H^{*} \leq H^{\prime \prime}, \quad \alpha^{\prime} \leq \alpha^{*} \leq \alpha^{\prime \prime}$ and $\delta_{\alpha}-$ the deviation of the angle $\alpha$ from this point. Then, by analogy with (5) for $H^{\prime}=0$ and $H^{\prime \prime}=H^{*}$ (Fig. 2)

$$
\begin{aligned}
& S_{1}^{*}=\int_{0}^{H^{*}}\left[B_{\|}\left(H, \alpha^{*}+\delta_{\alpha}\right)-B_{\|}\left(H, \alpha^{*}-\delta_{\alpha}\right)\right] \cdot \mathrm{d} H ; \\
& S_{2}^{*}=\int_{\alpha^{*}-\delta_{\alpha}}^{\alpha^{*}+\delta_{\alpha}}-H^{*} \cdot B_{\perp}\left(H^{*}, \alpha\right) \cdot \mathrm{d} \alpha .
\end{aligned}
$$

Limit expressions for these relations

$$
\begin{aligned}
& R_{1}^{*}=\lim _{\delta_{\alpha} \rightarrow 0} \int_{0}^{H^{*}} \frac{1}{2 \delta_{\alpha}}\left[B_{\|}\left(H, \alpha^{*}+\delta_{\alpha}\right)-B_{\|}\left(H, \alpha^{*}-\delta_{\alpha}\right)\right] \cdot \mathrm{d} H ; \\
& R_{2}^{*}=\lim _{\delta_{\alpha} \rightarrow 0} \int_{\alpha^{*}-\delta_{\alpha}}^{\alpha^{*}+\delta_{\alpha}} \frac{-1}{2 \delta_{\alpha}} H^{*} \cdot B_{\perp}\left(H^{*}, \alpha\right) \cdot \mathrm{d} \alpha .
\end{aligned}
$$

The first integral in (14) can be expressed in terms of the differential magnetic permeability $\mu_{\mathrm{d} H \alpha}$. The integral $R_{2}^{*}$, as can be seen from Fig. 2 , for $\delta_{\alpha} \rightarrow 0$, by analogy with (7), (8), degenerates into the line $c d$ :

$$
R_{1}^{*} \rightarrow c d=\int_{0}^{H_{1}^{*}} \mu_{\mathrm{d} H \alpha}\left(H, \alpha^{*}\right) \cdot \mathrm{d} H .
$$

Since for $H=0 H \cdot B_{\perp}=0$ for all $\alpha$, the value of the segment $c d$ determines the value of $H^{*} \cdot B_{\perp}\left(\boldsymbol{H}^{*}\right)$ and, therefore, $B_{\perp}\left(\boldsymbol{H}^{*}\right)$. Similarly, we can calculate the remaining components of the array $B_{\perp}(\boldsymbol{H})$.
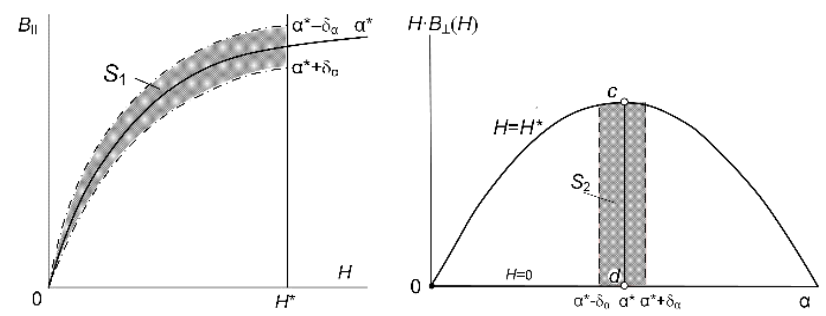

Fig. 2. Geometric meaning of the principle of reciprocity for polar coordinates

Computational experiments and discussion. In order to eliminate the instrumental error and ensure maximum accuracy and reliability of the results, we use the exact magnetization characteristics $B_{1}\left(H_{1}, H_{2}\right)$ and 
$B_{2}\left(H_{1}, H_{2}\right)$ obtained by differentiating the potential given in [8] (Fig. 3,a,b).

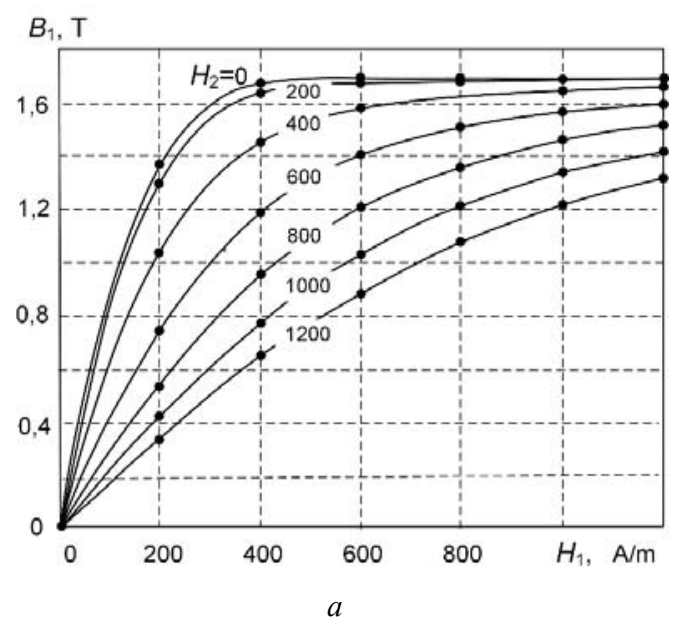

$B_{2}, T$

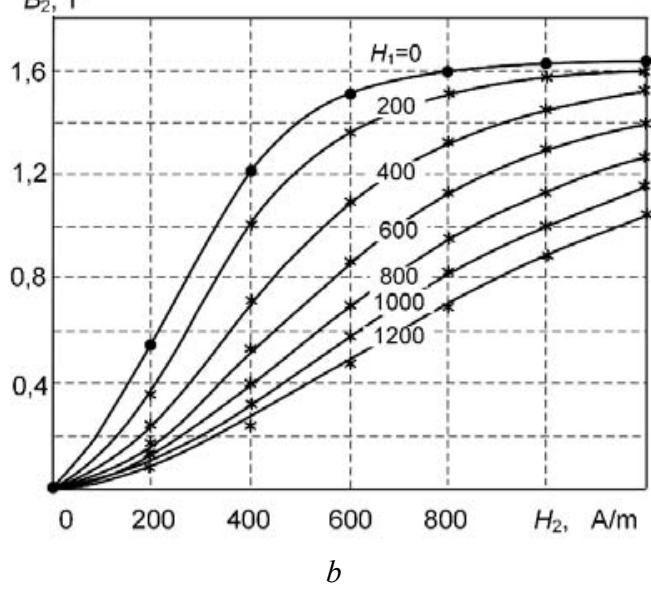

Fig. 3. Nonlinear anisotropic medium magnetization characteristics obtained by differentiation of the potential [8]

It follows from the reciprocity principle that the family of characteristics $B_{2}\left(H_{1}, H_{2}\right)$ can be calculated from the characteristics $B_{1}\left(H_{1}, H_{2}\right)$ and one of the characteristics $B_{2}\left(H_{1}, H_{2}\right)$, for example, $B_{2}\left(0, H_{2}\right)$. This information is reflected in Fig. 3,a,b by the symbols «॰ for nodes in which the values of magnetic flux density will be considered known. The symbols «*« correspond to the calculated values obtained by using expression (8) for the missing mesh nodes. From Fig. 3,b, one can see almost complete coincidence with the calculated dependences $B_{2}(\boldsymbol{H})$ (solid lines).

The algorithm of «filling» information on the magnetic properties of a nonlinear anisotropic medium is as follows. Using the known array of characteristics $B_{1}(\boldsymbol{H})$, by differentiation we obtain three components of the tensor $\boldsymbol{\mu}_{\mathrm{d}}(\boldsymbol{H}): \mu_{\mathrm{d} 11}(\boldsymbol{H})=$ $=\partial B_{1}(\boldsymbol{H}) / \partial H_{1} ; \mu_{\mathrm{d} 12}(\boldsymbol{H})=\partial B_{1}(\boldsymbol{H}) / \partial H_{2}=\mu_{\mathrm{d} 21}(\boldsymbol{H})$. Then, using expression (8), we find the corresponding values of the integrals $a b$, the subtraction of which from the values of the given characteristic $B_{2}\left(0, H_{2}\right)$ determines the family of characteristics $B_{2}(\boldsymbol{H})$ and, finally, $\mu_{\mathrm{d} 22}(\boldsymbol{H})=\partial B_{2}(\boldsymbol{H}) / \partial H_{2}$.

To confirm, we give some numerical examples. We choose two arbitrary vectors of magnetic field strength $\boldsymbol{H}$, for example, $\boldsymbol{H}^{(1)}=(450,600) \mathrm{A} / \mathrm{m}$ and $\boldsymbol{H}^{(2)}=(1120$, 375) $\mathrm{A} / \mathrm{m}$. The calculated values of the corresponding magnetic flux density vectors $\boldsymbol{B}^{(1)}=(1.259,1.005) \mathrm{T}$ and $\boldsymbol{B}^{(2)}=(1.669,0.275) \mathrm{T}$. And here, if the values of the $B_{1}$ components (1.259 $\mathrm{T}$ and $1.669 \mathrm{~T}$, respectively) are obtained by spline interpolation of a given array of nodal values of the magnetic flux density $B_{1}\left(H_{1}, H_{2}\right)$ (see Fig. 3,a), then the corresponding values of the $B_{2}$ components are calculated by the above technique without calculating the potential $\Psi$. The exact values of the magnetic flux density vectors obtained by using the analytical expressions from [8]: $\boldsymbol{B}^{(1)^{*}}=(1.259,1.022) \mathrm{T}$, $\boldsymbol{B}^{(2)^{*}}=(1.670,0.257) \mathrm{T}$. The mismatch angles between the vectors $\boldsymbol{B}$ and $\boldsymbol{H}$ are respectively equal $9.75^{\circ}$ and $14.07^{\circ}$.

We also give the calculated and exact $\left(^{*}\right)$ values of the differential absolute magnetic permeability tensors for given values $\boldsymbol{H}^{(1)}$ and $\boldsymbol{H}^{(2)}$ :

- for $\boldsymbol{H}=\boldsymbol{H}^{(1)}$

$$
\begin{aligned}
& \boldsymbol{\mu}_{\mathrm{d}}^{(1)}=\left|\begin{array}{ll}
\mu_{\mathrm{d} 11} & \mu_{\mathrm{d} 12} \\
\mu_{\mathrm{d} 21} & \mu_{\mathrm{d} 22}
\end{array}\right|=10^{-3} \cdot\left|\begin{array}{rr}
1.28247 & -1.25014 \\
-1.25014 & 1.54950
\end{array}\right| \text {, } \\
& \mu_{\mathrm{d}}^{(1)^{*}}=10^{-3} \cdot\left|\begin{array}{rr}
1.26986 & -1.23883 \\
-1.23883 & 1.56906
\end{array}\right| \text {; }
\end{aligned}
$$

- for $\boldsymbol{H}=\boldsymbol{H}^{(2)}$

$$
\begin{aligned}
& \boldsymbol{\mu}_{\mathrm{d}}^{(2)}=10^{-4} \cdot\left|\begin{array}{rr}
0.53929 & -2.19504 \\
-2.19504 & 10.7357
\end{array}\right|, \\
& \boldsymbol{\mu}_{\mathrm{d}}^{(2)^{*}}=10^{-4} \cdot\left|\begin{array}{cc}
0.53756 & -2.21087 \\
-2.21087 & 11.2446
\end{array}\right| .
\end{aligned}
$$

We note that the given values of the tensors $\boldsymbol{\mu}_{\mathrm{d}}(\boldsymbol{H})$ are local, therefore, from the fact that for the selected values of the field strength vectors $\mu_{\mathrm{d} 22}>\mu_{\mathrm{d} 11}$, it cannot be concluded that the $\mathrm{H}_{2}$ axis is the direction of easy magnetization. As will be illustrated in Fig. 4, this direction is the axis $H_{1}$.

We also note one of the useful consequences of the integral reciprocity principle: at the same scales for the corresponding components of the vectors $\boldsymbol{B}$ and $\boldsymbol{H}$, the areas bounded by the limiting magnetization curves are the same, since according to (4) the areas of all the corresponding curved quadrangles are the same. For shown in Fig. 3, $a, b$ boundary characteristics $B_{1}\left(H_{1}, 0\right)$ and $B_{1}\left(H_{1}, 1200\right), B_{2}\left(0, H_{2}\right)$ and $B_{2}\left(1200, H_{2}\right)$, by integration almost identical values were obtained: $880.4670 \mathrm{~J}$ and $880.4688 \mathrm{~J}$. This property can be useful in conditions of limited information on the magnetic properties of an anisotropic medium, when only two characteristics are specified in orthogonal directions.

To further illustrate the anisotropic properties of the considered medium, Fig. 4 shows the hodographs of the vector $\boldsymbol{H}$ (semicircles of radii of 1200,600 , and $300 \mathrm{~A} / \mathrm{m}$ ) and the corresponding hodographs of the magnetic flux density vector $\boldsymbol{B}$. The anisotropy of the medium is manifested by a pronounced nonlinear dependence of magnetic flux density on the field strength, more «easy» magnetization in the direction of the $H_{1}$ axis, and significant mismatch between vectors $\boldsymbol{B}$ and $\boldsymbol{H}$ in almost the entire range of field changes. 


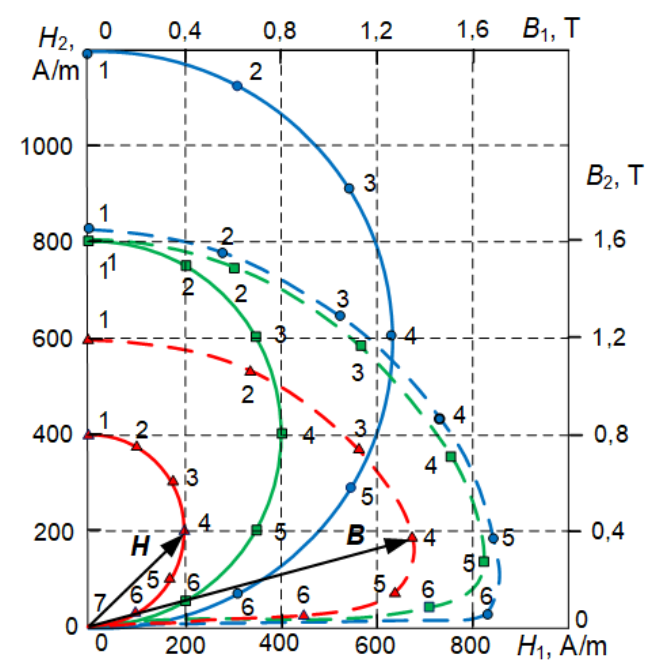

Fig. 4. Hodographs of the change of vectors $\boldsymbol{B}$ and $\boldsymbol{H}$ of a nonlinear anisotropic medium

Figures 5,a,b show the magnetization characteristics for the polar coordinate system $(H, \alpha)$, which are obtained by recalculating characteristics presented in Fig. 3, $a, b$. As in Fig. 3, the symbols «॰» mark the nodes of the «longitudinal» magnetization characteristics with known magnetic flux density values at $H=0: 200: 1200 \mathrm{~A} / \mathrm{m}$, $\alpha=0: \pi / 12: \pi / 2$, and the symbols $《 *\rangle$ correspond to the calculated values obtained using the expression (15) for nodes of the «transverse» magnetization characteristics $B_{\perp}(H, \alpha)$.
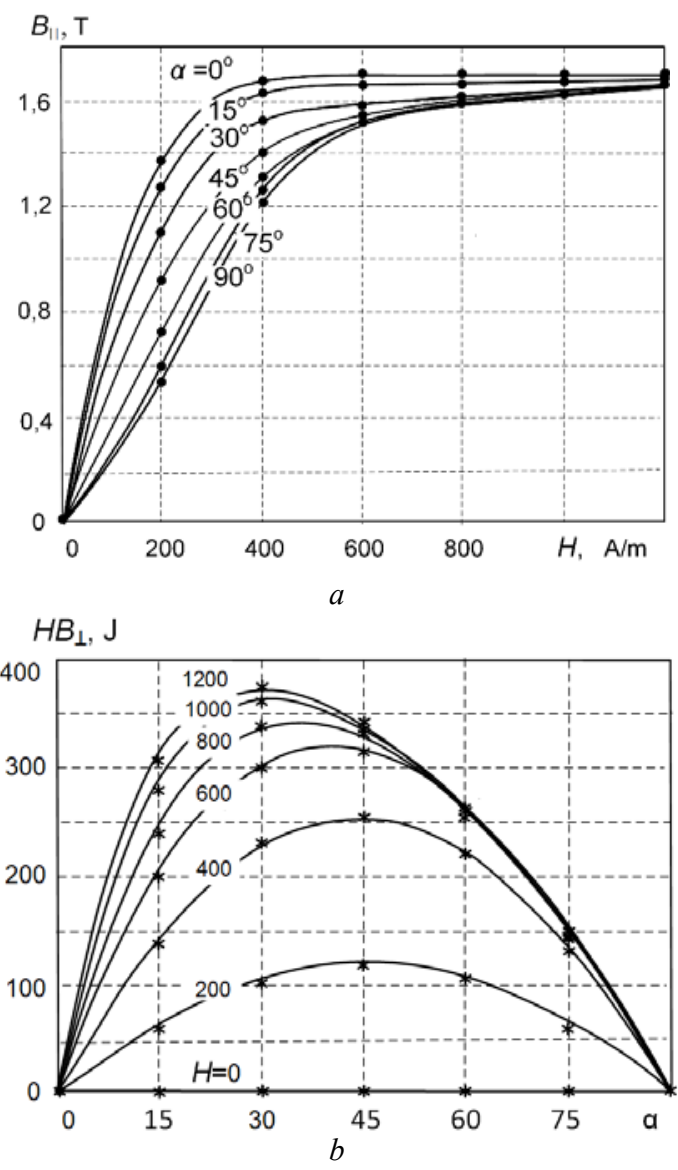

Fig. 5. «Longitudinal» $-a$ and «transverse» $-b$ characteristics of magnetization of an anisotropic medium obtained by recalculation of characteristics in Fig. 3
The calculation algorithm for the given characteristics $B_{\|}(H, \alpha)$ of the magnetization in the orthogonal direction $B_{\perp}(H, \alpha)$ and the differential magnetic permeability tensor $\boldsymbol{\mu}_{\mathrm{d}}(\boldsymbol{H})$ remains almost the same as in the case of Cartesian coordinates described above: according to the characteristics $B_{\|}(H, \alpha)$, we first determine the three components of the tensor $\boldsymbol{\mu}_{\mathrm{d}}(\boldsymbol{H}): \mu_{\mathrm{d} H H}=\partial B_{\|} / \partial H, \mu_{\mathrm{d} H \alpha}=$ $=\frac{1}{H} \partial B_{\|} / \partial \alpha=\mu_{\mathrm{d} \alpha H}=\frac{1}{H} \partial\left(H \cdot B_{\perp}\right) / \partial H$. Then, by integration according to expression (15), we find the values $H \cdot B_{\perp}(H, \alpha)$ for all $H=$ const and, finally, the values of the missing component $\mu_{\mathrm{d} \alpha \alpha}=\frac{1}{H} \partial B_{\perp} / \partial \alpha$.

The validity of the obtained results is confirmed by numerical calculations. For previously accepted values of the magnetic field strength vector $\boldsymbol{H}^{(1)}=(450,600) \mathrm{A} / \mathrm{m}=$ $=(H, \alpha)=\left(750 \mathrm{~A} / \mathrm{m}, 39.06^{\circ}\right)$ and $\boldsymbol{H}^{(2)}=(1120,375) \mathrm{A} / \mathrm{m}=$ $=\left(1181 \mathrm{~A} / \mathrm{m}, 8.758^{\circ}\right)$ calculated values of the corresponding magnetic flux density vectors $\boldsymbol{B}^{(1)}=$ $=\left(B_{\|}, B_{\perp}\right)=(1.574,0.392) \mathrm{T}$ and $\boldsymbol{B}^{(2)}=(1.666,0.283) \mathrm{T}$. Exact values of magnetic flux density vectors obtained using analytical formulas from [8]: $\boldsymbol{B}^{(1)^{*}}=(1.573,0.394) \mathrm{T}$, $\boldsymbol{B}^{(2)^{*}}=(1.666,0.286) \mathrm{T}$.

In conclusion, we note that the integrand in the integral (1) characterizes the change in the density of the co-energy of the magnetic field spent on the cyclic magnetization of the medium. The results obtained can easily be transferred to a similar integral for the energy density $\boldsymbol{H} \mathrm{d} \boldsymbol{B}$, the use of which leads to the vector dependencies $\boldsymbol{H}(\boldsymbol{B})$, namely $H_{1}\left(B_{1}, B_{2}\right)$ and $H_{2}\left(B_{1}, B_{2}\right)$ or $H_{\|}(B, \alpha)$ and $H_{\perp}(B, \alpha)$ depending on the selected coordinate system.

Examples of the use of the results obtained in relation to anisotropic electrical steels will be the subject of special consideration.

\section{Conclusions.}

1. The task of constructing the correct vector material equations for nonlinear anisotropic soft magnetic materials remains one of the main reserves for increasing the accuracy of mathematical models in solving magnetostatic problems in the field formulation.

2. An effective direction for solving this problem, which has been actively developing in recent years, is to use the energy approach to constructing the vector characteristics of magnetization. However, the impossibility of directly measuring energy potentials, the complexity of the analytical description and ensuring accuracy with double differentiation to determine the differential magnetic permeability tensor make the task of constructing them quite time-consuming.

3. An alternative technique of constructing the vector characteristics of magnetization is to use the reciprocity principle, which is valid for media with reversible magnetization processes. Its main advantage is the ability to directly recalculate the magnetization characteristics in one of the directions according to the specified magnetization characteristics in the orthogonal direction without calculating the energy potential. 
4. The asymptotic expressions for the reciprocity principle established in this paper, which are universal in character for arbitrary magnetic media in the hysteresisfree approximation, open up additional possibilities for optimizing computational processes and increasing the accuracy of numerical methods for solving magnetostatic problems in the field formulation.

\section{REFERENCES}

1. Silvester P.P., Gupta R.P. Effective computational models for anisotropic soft B-H curves. IEEE Transactions on Magnetics, 1991, vol. 27, no. 5, pp. 3804-3807. doi: 10.1109/20.104930.

2. Liu J., Basak A., Moses A.J., Shirkoohi G.H. A method of anisotropic steel modelling using finite element method with confirmation by experimental results. IEEE Transactions on Magnetics, 1994, vol. 30, no. 5, pp. 3391-3394. doi: 10.1109/20.312666.

3. Lin D., Zhou P., Badics Z., Fu W.N., Chen Q.M., Cendes Z.J. A new nonlinear anisotropic model for soft magnetic materials. IEEE Transactions on Magnetics, 2006, vol. 42, no. 4, pp. 963-966. doi: 10.1109/TMAG.2006.871380.

4. Sabonnadiere J.C., Meunier G. Modelling nonlinear magnetic materials for field computation. Journal of Electromagnetic Waves and Applications, 1990, no. 4, pp. 10271054.

5. Bíró O., Außerhofer S., Preis K., Chen Y. A modified elliptic model of anisotropy in nonlinear magnetic materials. COMPEL - The international journal for computation and mathematics in electrical and electronic engineering, 2010, vol. 29, no. 6, pp. 1482-1492. doi: 10.1108/03321641011078553.

6. Higuchi S., Nakao T., Takahashi Y., Tokumasu T., Fujiwara K., Ishihara Y. Modeling of two-dimensional magnetic properties based on one-dimensional magnetic measurements. IEEE Transactions on Magnetics, 2012, vol. 48, no. 11, pp. 3486-3489. doi: 10.1109/TMAG.2012.2198878.

7. Martin F., Singh D., Belahcen A., Rasilo P., Haavisto A., Arkkio A. Analytical model for magnetic anisotropy of non- oriented steel sheets. COMPEL - The international journal for computation and mathematics in electrical and electronic engineering, 2015, vol. 34, no. 5, pp. 1475-1488. doi: 10.1108/compel-02-2015-0076.

8. Tolmachev S., Il'chenko O. Mathematical modelling of magnatic properties of non-linear anisotropic medium in anhysteretic approximation. Proc. of International Conference Modern electrical and energy systems (MEES 2017), November 15-17, 2017, Kremenchuk, Ukraine, pp. 316-319. doi: 10.1109/MEES.2017.8248920.

9. Tolmachev S.T., Rozhnenko Z.G. The reciprocity principle for a magnetic medium without hysteresis. Elektrichestvo, 1992, no. 12 , pp. 51-53. (Rus).

10. Tolmachev S., Rozhnenko Z. The theory of the defining equations for nonlinear anisotropic materials. Proc. of the XIII International Symposium on Theoretical Electrical Engineering ISTET'05, Ukraine, Lviv, 2005, pp. 97-100.

11. Maergoiz I.D. Iteratsionnyye metody rascheta staticheskikh poley $v$ neodno-rodnykh, anizotropnykh i nelineynykh sredakh. [Iterative methods for calculating static fields in inhomogeneous anisotropic and nonlinear media]. Kiev, Naukova Dumka Publ., 1979. 210 p. (Rus).

12. Bortakovsky A.S., Panteleev A.V. Lineynaya algebra $v$ primerakh i zadachakh: Uchebnoye posobiye. [Linear algebra in examples and tasks]. Moscow, Vysshaya shkola Publ., 2005. 509 p. (Rus).

Received 29.10.2019

S.T. Tolmachev ${ }^{1}$, Doctor of Technical Science, Professor, A.V. Il'chenko ${ }^{1}$, Candidate of Technical Science, Associate Professor,

${ }^{1}$ Kryvyi Rih National University,

11, Vitaly Matusevich Str., Kryvyi Rih, Dnipropetrovsk Region, 50027, Ukraine,

e-mail: kafem.knu@gmail.com

How to cite this article:

Tolmachev S.T., Il'chenko A.V. The reciprocity principle for a nonlinear anisotropic medium without hysteresis: theory and practice of application. Electrical engineering \& electromechanics, 2020, no.2, pp. 40-45. doi: 10.20998/2074272X.2020.2.06. 